\title{
Nurse rostering: models and algorithms for theory, practice and integration with other problems
}

\author{
Pieter Smet
}

Received: date / Accepted: date

This is a summary of the author's PhD thesis supervised by Patrick De Causmaecker and Greet Vanden Berghe and defended on July 3, 2015 at KU Leuven. The thesis is written in English and is available from the author upon request at pieter.smet@cs.kuleuven.be and from http://lirias.kuleuven. be/handle/123456789/496913.

This work concerns nurse rostering, an operational personnel scheduling problem in health care in which the goal is to assign shifts to nurses, thereby satisfying staffing demands. Rostering problems are typically subject to a wide variety of constraints regarding personal preferences, organisational guidelines and labour legislation. While nurse rostering is considered a well-established operational research field, this thesis identifies a set of unexplored open problems and contributes both theoretical and practical results. Note that while the title and text of this thesis explicitly refers to nurse rostering, the mathematical models and algorithms that were created are not restricted to this application, but are useful in a variety of other settings where staff rosters must be created.

This dissertation contributes significant theoretical results which provide crucial insight for academic personnel rostering. Several polynomially solvable rostering problems are identified by constructing new minimum cost network flow formulations. By situating these easy rostering problems within existing academic literature, considerable insight is provided into precisely what makes personnel rostering hard. This dissertation further challenges the strong simplifications concerning constraint evaluation at the boundaries of rostering horizons commonplace in most previous research. After identifying the inaccuracies and quantifying the risks of this simplification, a methodology is provided for consistently evaluating personnel rostering solutions across consecutive rostering horizons. 
In addition to its theoretical results, this dissertation presents several contributions which significantly improve practical decision support systems for rostering, thereby abridging the gap between research and practice. To this end, a rich model for practical rostering problems is proposed, aimed at capturing the complex characteristics arising from practical rostering environments. To stimulate research on these challenging problems, a new benchmark dataset based on real world data has been made publicly available. In addition to the model, a methodology is developed which automatically learns each planner's preferences, thereby significantly facilitating the practical implementation of decision support systems for rostering. These two contributions have been successfully integrated into a commercial personnel rostering software system and are currently used in a variety of hospitals and other organisations across Europe.

Finally, this dissertation generalises shift-based demand models for rostering by including task scheduling as an additional decision. Three problem variants of the resulting integrated personnel rostering and task scheduling problems are studied. By utilising new hybrid heuristic algorithms which combine heuristic search and mathematical programming techniques such as integer programming and column generation, state of the art results are obtained. Extensive computational experiments demonstrate the behaviour of the proposed algorithms and investigate the empirical hardness of one of the problem variations. 